\title{
Predictive control of networked control systems based on event-triggered
}

\author{
Wangzhi Wen ${ }^{1, a}$, Zhongmeng Xiao $^{2, b}$ \\ ${ }^{1}$ Department of Electrical and Information Engineering, Lanzhou University of Technology, Lanzhou, \\ 730050, China \\ ${ }^{2}$ Department of Electrical and Information Engineering, Lanzhou University of Technology, Lanzhou, \\ 730050, China \\ aemail: wwwangzhiwen@163.com, bemail: zhongmengxiao2014@163.com
}

Keywords: Event-triggered; Predictive Control; Output Feedback; Packet Loss; LMI

\begin{abstract}
A novel structure of networked control systems which incorporates output feedback predictive control and event-triggered communication scheme in a unified model based on the networked control system with packet loss is proposed in this paper. The load of communication channel is relieved by event-triggered communication scheme, and the packet loss is compensated actively by predictive control. Then, the stability criterion which can guarantee the asymptotic stability of the closed-loop system is established and output feedback controller gain is obtained using the approach of a common quadratic Lyapunov and linear matrix inequality based on this model. A simulation example is given to demonstrate the effectiveness of the proposed method. The whole improvement of quality of performance and network quality of service is realized.
\end{abstract}

\section{Introduction}

Networked Control Systems (NCSs) are syncretic products of modern control technology and computer communication technology which has brought them new opportunities and challenges. In recent years, the analysis and synthesis of networked control systems with network-induced delay and data packet loss has become a research focus in the international academic circles.

Uncertainties of network such as network-induced delay and data dropout are inevitable because sampling signals and control signals are transmitted through network in the network control systems, and all sampling signals will be sent by traditional way of sampling. So the network congestion will be caused and then packet loss will be happened easily. The network data transmission quantity can be reduced effectively by event-triggered communication scheme, and the load of communication channel will be relieved and the packet loss will be reduced afterwards. There are a lot of fruitful literatures at home and abroad in the respect ${ }^{[1-3]}$. In [4], a distributed event-triggered scheme was proposed taking network-induced delay and packet loss into account. Networked predictive control method is a kind of prediction compensation algorithm based on a model in NCSs. Because the network induced delay and packet loss can be compensated actively by the algorithm, some scholars combined event-triggered scheme and predictive control to research. A networked predictive control method was proposed to compensate delay and data dropout actively in [5-6]. It is verified by experimental results that networked predictive control method can obtain a similar performance as local control. In [7], a novel event-triggered networked predictive control method was designed which decided controlled variable according to the output of object rather than the network delay.

A novel structure of networked control systems which incorporates output feedback predictive control and event-triggered communication scheme in a unified model is proposed in this paper. The load of communication channel can be relieved by the event-triggered communication scheme. Although the network bandwidth resource is saved by the scheme, the quality of control will be reduced at the same time. The predictive control algorithm can compensate packet loss in advance. The two aspects split the difference, and the ideal quality of performance and quality of service can be obtained. Then, the stability criterion which can guarantee the asymptotic stability of the 
closed-loop system is established and output feedback controller gain is obtained using the approach of a common quadratic Lyapunov and linear matrix inequality based on this model. A simulation example is given to demonstrate the effectiveness of the proposed method.

\section{Problem Description}

\section{Model of system}

The structure of networked control system is shown in Figure 1. The dynamic equation of the plant and model are described respectively by the following discrete-time linear systems:

$$
\begin{aligned}
& \left\{\begin{array}{l}
x\left(t_{k}+1\right)=A x\left(t_{k}\right)+B u\left(t_{k}\right) \\
y\left(t_{k}\right)=C x\left(t_{k}\right)
\end{array}\right. \\
& \left\{\begin{array}{l}
\hat{x}\left(t_{k}+1\right)=\hat{A} \hat{x}\left(t_{k}\right)+\hat{B} u\left(t_{k}\right) \\
\hat{y}\left(t_{k}\right)=\hat{C} \hat{x}\left(t_{k}\right)
\end{array}\right.
\end{aligned}
$$

Where $x\left(t_{k}\right) \in \mathbb{R}^{n}$ and $\hat{x}\left(t_{k}\right) \in \mathbb{R}^{n}, u\left(t_{k}\right) \in \mathbb{R}^{m}, \quad y\left(t_{k}\right) \in \mathbb{R}^{r}$ and $\hat{y}\left(t_{k}\right) \in \mathbb{R}^{r}$ denote the state vectors, control vectors and output vectors. The output feedback control $u(t)=K \hat{y}$ is adopted, $K$ is the output feedback matrix. $A, B$ and $C$ are constant system matrices with appropriate dimensions. $\hat{A}, \hat{B}$ and $\hat{C}$ are the model of system matrices $A, B$ and $C$. Assuming the model is accurate, that is $A=\hat{A}, B=\hat{B}, C=\hat{C}$. The initial condition of system (1) is given by $x\left(t_{0}\right)=x_{0}$.

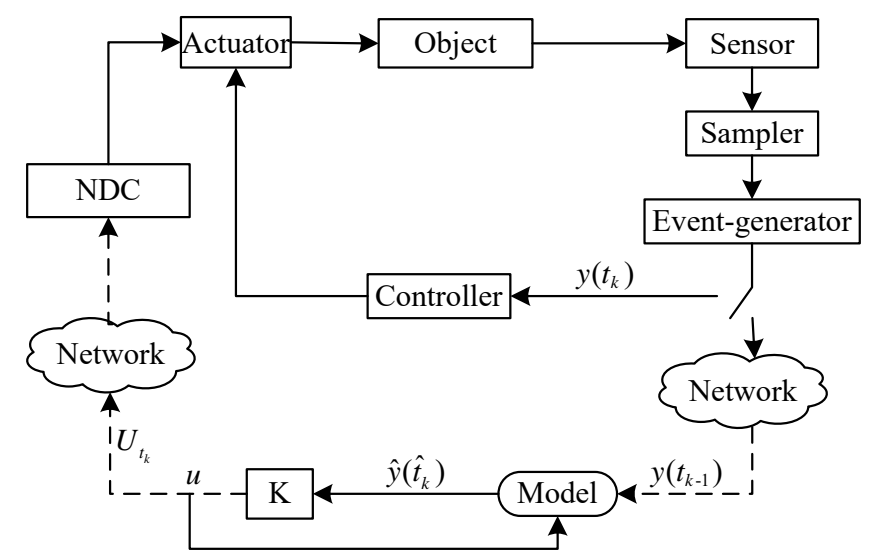

Fig.1. Structure of the event-triggered predictive control system

A certain discrete network control system is considered in the paper. The event-triggered scheme and output feedback predictive control can save network bandwidth and compensate packet loss actively to ensure whole improvement of control quality of performance and network quality of service. This scheme mainly includes three parts: event-generator at the sensor side, controller prediction generator(CPG) at the controller side and the networked delay compensator(NDC) at the actuator side.

Before further describing the scheme, we make the following assumptions:

Assumption 1. The sensor is time-driven with a constant sampling period T. The controller and actuator are event-driven. This paper assumes that the network time delay does not exist.

Assumption 2. The upper bound of continuous packet loss from sensor to actuator is $p, p$ is a positive integer.

\section{Event-triggered predictive control scheme}

Discrete event-triggered communication scheme refers to introducing a communication constraint to NCSs. It transmits information according to whether the state and state error satisfy the condition or not. State of the system is detected at each sampling instant and corresponding error is calculated. If it meets the triggering condition, the current state of the system is transmitted. On the assumption that the latest sampling data is $y\left(t_{k}\right)$, the current sampling data is $y\left(i_{k}\right)$. The following 
triggering condition is established from the viewpoint of saving network resources:

$$
\left[y\left(i_{k}\right)-y\left(t_{k}\right)\right]^{T} \Phi\left[y\left(i_{k}\right)-y\left(t_{k}\right)\right] \geq \mu y^{T}\left(i_{k}\right) \Phi y\left(i_{k}\right)
$$

where $\Phi$ is event-triggered matrix, $\mu$ is a constant. For the sake of facilitate analysis, the state error vector is defined as follows:

$$
e\left(i_{k}\right)=y\left(i_{k}\right)-y\left(t_{k}\right)
$$

The event-triggered communication condition can be written as the following inequality:

$e^{T}\left(i_{k}\right) \Phi e\left(i_{k}\right) \geq \mu y^{T}\left(i_{k}\right) \Phi y\left(i_{k}\right)$

If the current output $y\left(t_{k}\right)$ is transmitted at time $t_{k}$, the next event-triggered instant is determined by the following condition:

$$
t_{k+1}=t_{k}+\min _{r}\left\{r \mid\left[y\left(t_{k}+r\right)-y\left(t_{k}\right)\right]^{T} \Phi\left[y\left(t_{k}+r\right)-y\left(t_{k}\right)\right]>\mu y\left(t_{k}+r\right)^{T} \Phi y\left(t_{k}+r\right)\right\}
$$

where $k=1,2, \ldots, 0<\mu<1$ is a given constant, $\Phi$ is a positive definite weighting matrix to be designed. It can be seen from the event-triggered scheme (3) that the next event-triggered moment $t_{k+1}$ not only depends on the error of current sampling data and the latest sampling data, but also depends on the current sampling data $y\left(t_{k}+r\right)(r=1,2, \ldots)$ and the constant $\mu$ and matrix $\Phi$. At the sensor side, only part of the measurement information needs to be sent to the controller side. Therefore, the event-triggered scheme (3) can reduce the information transmission between the sensor and controller. In view of the packet loss is inevitable in actual communication network, so not all of the triggered output values $\left\{y\left(t_{k}\right)\right\}_{k=1}^{\infty}$ will be transmitted to the controller side successfully. So as to take packet loss account into the model of NCSs, $\left\{y\left(t_{s i}\right)\right\}_{i=1}^{\infty}$ defines the triggered values which are transmitted successfully. It must be pointed out that successful transmitting means $y\left(t_{s i}\right)$ is transmitted from the sensor side to the controller side and corresponding prediction sequence package is transmitted to the actuator side successfully. Apparently, $\quad\left\{y\left(t_{s i}\right)\right\}_{i=1}^{\infty} \subseteq\left\{y\left(t_{k}\right)\right\}_{k=1}^{\infty} \cdot T_{s i}=\left\{\hat{t}_{s i+1}, \hat{t}_{s i+2}, \ldots, \hat{t}_{s i+p}\right\}\left(t_{s i}<\hat{t}_{s i+1}<\hat{t}_{s i+2}<\ldots<\hat{t}_{s i+p}\right)$ is the set of predictive event-triggered instant by event generator, which are determined by the following event-triggering condition:

$$
\begin{aligned}
\hat{t}_{s_{i}+1} & =t_{s_{i}}+\min _{r}\left\{r \mid\left[\hat{y}\left(t_{s_{i}}+r\right)-y\left(t_{s_{i}}\right)\right]^{T} \Phi\left[\hat{y}\left(t_{s_{i}}+r\right)-y\left(t_{s_{i}}\right)\right]>\mu \hat{y}\left(t_{s_{i}}+r\right)^{T} \Phi \hat{y}\left(t_{s_{i}}+r\right)\right\} \\
\hat{t}_{s_{i}+m+1} & =\hat{t}_{s_{i+m}}+\min _{r}\left\{r \mid\left[\hat{y}\left(\hat{t}_{s_{i}+m}+r\right)-\hat{y}\left(\hat{t}_{s_{i+m}}\right)\right]^{T} \Phi\left[\hat{y}\left(\hat{t}_{s_{i+m}}+r\right)-\hat{y}\left(\hat{t}_{s_{i+m}}\right)\right]>\mu \hat{y}\left(\hat{t}_{s_{i+m}}+r\right)^{T} \Phi \hat{y}\left(\hat{t}_{s_{i+m}}+r\right)\right\} \\
& i \in\{1,2, \ldots, \infty\}, m \in\{1,2, \ldots, p-1\}
\end{aligned}
$$

where $\mu$ and $\Phi$ are the same as that in Eq. (3).

$u\left(t_{k}\right)=K y\left(t_{k}\right)=K C x\left(t_{k}\right), \quad \hat{x}\left(t_{k}+1\right)=A x\left(t_{k}\right)+B K C x\left(t_{k}\right), \quad \hat{x}\left(\hat{t}_{s_{1}+1}\right)$ is the first event-triggered predictive state based on the state $x\left(t_{s i}\right) \cdot \hat{x}\left(\hat{t}_{s i+m}\right)$ is the subsequent event-triggered predictive state which can be calculated iteratively:

$$
\begin{aligned}
\hat{x}\left(t_{s_{i}}+1\right) & =A x\left(t_{s_{i}}\right)+B K C x\left(t_{s_{i}}\right) \\
\hat{x}\left(t_{s_{i}}+j+1\right) & =A \hat{x}\left(t_{s_{i}}+j\right)+B K C x\left(t_{s_{i}}\right) \\
j & =1,2, \ldots, \hat{t}_{s_{i}+1}-t_{s_{i}}-1 \\
& \vdots \\
\hat{x}\left(\hat{t}_{s_{i}+m}+j+1\right) & =A \hat{x}\left(\hat{t}_{s_{i}+m}+j\right)+B K C \hat{x}\left(\hat{t}_{s_{i}+m}\right) \\
j & \in\left\{0,1,2, \ldots, \hat{t}_{s_{i}+m+1}-\hat{t}_{s_{i}+m}-1\right\} \\
m & \in\{1,2, \ldots, p-1\}
\end{aligned}
$$

At each $t_{s i}$, based on the obtained states $x\left(t_{s i}\right), \hat{x}\left(\hat{t}_{s i+1}\right), \ldots, \hat{x}\left(\hat{t}_{s+p}\right)$, corresponding predictive controller laws are given by

$$
\begin{aligned}
u\left(t_{s_{i}}\right) & =K C x\left(t_{s_{i}}\right) \\
\hat{u}\left(\hat{t}_{s_{i+1}}\right) & =K C \hat{x}\left(\hat{t}_{s_{i+1}}\right) \\
& \vdots \\
\hat{u}\left(\hat{t}_{s_{i+p}}\right) & =K C \hat{x}\left(\hat{t}_{s_{i}+p}\right)
\end{aligned}
$$


At the controller prediction generator, the control sequence above is packetized as a single packet denoted by

$$
U_{t_{i j}} \triangleq\left[u\left(t_{s i}\right), \hat{u}\left(\hat{t}_{s+1}\right), \hat{u}\left(\hat{t}_{s i+2}\right), \cdots, \hat{u}\left(\hat{t}_{s i+p}\right)\right]
$$

and then sent it to the actuator side via communication network at time $t_{s i}$. At each instant $t_{s i}$, $U_{t_{s i-1}}$ at the actuator side is discarded and replaced by $U_{t_{i j}}$. For $t \in\left[t_{s i}, t_{s_{i+1}}\right)$, the NDC chooses the suitable control signal from $U_{t_{i}}$ to act on the plant until a new control packet $U_{t_{s_{i+1}}}$ is received by the actuator. In fact, at each $t_{s i+j} \in\left[t_{s i}, t_{s i+1}\right)$, if $x\left(t_{s i+j}\right)$ is released at the sensor node, then the corresponding control signal $\hat{u}\left(\hat{t}_{s_{i}+j}\right)$ in $U_{t_{i}}$ will be used to determine the plant's next triggered state $x\left(t_{s_{i+j+1}}\right), j \in\left\{1,2, \ldots, s_{i+1}-s_{i}-1\right\}$. Because of $\hat{A}=A, \quad \hat{B}=B, \quad C=\hat{C}$, the relations $x\left(t_{s i+j}\right)=\hat{x}\left(t_{s i+j}\right)$ and $t_{s i+j}=\hat{t}_{s i+j}, j \in\left\{1,2, \ldots, s_{i+1}-s_{i}-1\right\}$ are true which will be clearly shown below.

Lemma $10^{[8]}$ After time $t_{\mathrm{s},}$, for $\forall t_{k} \in\left[t_{\mathrm{s} \mid}+1, \infty\right)$, the state $x(t)$ of system (1) and the state $x(t)$ of the predictive system (5) have the following relationship:

$$
x\left(t_{k}\right)=\hat{x}\left(t_{k}\right), t_{k} \in\left[t_{s 1}+1, \infty\right)
$$

which implies that $x\left(t_{s_{i+j}}\right)=\hat{x}\left(\hat{t}_{s_{i+j}}\right)$ and $t_{s^{i}+j}=\hat{t}_{s^{i}+j}, j \in\left\{1,2, \ldots, S_{i+1}-s_{i}-1, i=1,2, \ldots\right\}$. The proof is omitted.

Remark 1. For $t \in\left[t_{0}, t_{\mathrm{s} 1}\right)$, the plant is working with zero control, so $x\left(t_{\mathrm{sl}}\right)=A^{t_{\mathrm{s} 1}-1} x\left(t_{0}\right)$.

\section{Stability analysis and controller design}

According to Lemma (1), system (1) under the event-triggered condition (3) can be transformed into the following closed-loop system considering packet loss compensation:

$$
\begin{aligned}
x\left(t_{k}+1\right) & =A x\left(t_{k}\right)+B K C \hat{x}\left(\hat{t}_{s_{i}+j}\right) \\
& =A x\left(t_{k}\right)+B K C x\left(t_{s_{i}+j}\right) \\
x\left(t_{s_{1}}\right) & =A^{t_{s_{1}}-t_{0}} x\left(t_{0}\right) \\
t_{k} & \in\left[t_{s_{i}}, t_{s_{i+1}}\right) \bigcap\left[t_{s_{i}+j}, t_{s_{i}+j+1}\right)
\end{aligned}
$$

where $x\left(t_{s 1}\right)$ is the initial condition.

Define the error vector $e_{i j}(t)=y\left(t_{k}\right)-y\left(t_{s i+j}\right)$ for $t \in\left[t_{s i}, t_{s i+1}\right) \cap\left[t_{s i+j}, t_{s_{i}+j+1}\right)$. Then the closed-loop system (6) can be written as

$$
\begin{aligned}
x\left(t_{k}+1\right) & =A x\left(t_{k}\right)+B K C x\left(t_{k}\right)-B K e_{i j}(t) \\
t_{k} & \in\left[t_{s_{i}}, t_{s_{i+1}}\right) \cap\left[t_{s_{i}+j}, t_{s_{i}+j+1}\right) \\
j & =0,1,2, \ldots, S_{i+1}-s_{i}-1 \\
x\left(t_{s_{1}}\right) & =A^{t_{s_{1}}-t_{0}} x\left(t_{0}\right)
\end{aligned}
$$

Remark 2. Note that for $t \in\left[t_{s i}, t_{s_{i+1}}\right) \cap\left[t_{s_{i+j}}, t_{s_{i+j+1}}\right)$, no event is triggered on the sensor node, so based on event-triggered condition (3), there is

$$
e_{i j}^{T}(t) \Phi e_{i j}(t) \leq \mu y^{T}(t) \Phi y(t)
$$

It can be seen that $\left[t_{s i}, \infty\right)=\bigcup_{i=1}^{\infty} \bigcup_{j=0}^{s_{i+1}-s_{i-1}}\left\{\left[t_{s_{i}}, t_{s_{i+1}}\right) \bigcap\left[t_{s_{i+j}}, t_{s_{i+j+1}}\right)\right\}$.

Theorem 1. Under assumptions (1)-(2), for given system matrices $A, B$ and $C$ and $a$ constant parameter $0<\mu<1$, the system described by (7) is asymptotically stable if there exist matrices $\tilde{P}>0, \tilde{\Phi}>0$ and $Y$ with appropriate dimensions which make the following LMI is satisfied:

$$
\left[\begin{array}{lll}
-\tilde{P}+\mu \tilde{\Phi} & * & * \\
0 & -\tilde{\Phi} & * \\
A \tilde{P}+B Y & -B Y & -\tilde{P}
\end{array}\right]<0
$$


with

$\Phi=C^{-1} \tilde{P} \tilde{P}^{-1} \tilde{\Phi} \tilde{P}^{-1} C^{-1}, K=Y \tilde{P}^{-1} C^{-1}$

Proof. Construct the following Lyapunov function as

$V(t)=x^{T}(t) P x(t)$

where $P$ is a symmetric positive definite matrix. For $t \in\left[t_{s i}, t_{s i+1}\right) \cap\left[t_{s i+j}, t_{s i+j+1}\right)$, along the trajectory of system (7) and taking Eq. (8) into account, the forward difference of $V(t)$ is given by

$$
\begin{aligned}
& \Delta V(t)=V(t+1)-V(t) \\
& \leq x^{T}(t+1) P x(t+1)-x^{T}(t) P x(t)-\left[e_{i j}{ }^{T}(t) \Phi e_{i j}(t)-\mu x^{T}(t) C^{T} \Phi C x(t)\right] \\
& =\left[A x(t)+B K C x(t)-B K e_{i j}(t)\right]^{T} P\left[A x(t)+B K C x(t)-B K e_{i j}(t)\right] \\
& -x^{T}(t) P x(t)-e_{i j}^{T}(t) \Phi e_{i j}(t)+\mu x^{T}(t) C^{T} \Phi C x(t) \\
& =\left[\begin{array}{ll}
x^{T}(t) & e_{i j}^{T}(t)
\end{array}\right] \Omega\left[\begin{array}{c}
x(t) \\
e_{i j}(t)
\end{array}\right]
\end{aligned}
$$

where

$$
\begin{aligned}
& \Omega=\left[\begin{array}{cc}
(A+B K C)^{T} P(A+B K C)-P+\mu C^{T} \Phi C & * \\
-(B K)^{T} P(A+B K C) & (B K)^{T} P B K-\Phi
\end{array}\right]
\end{aligned}
$$

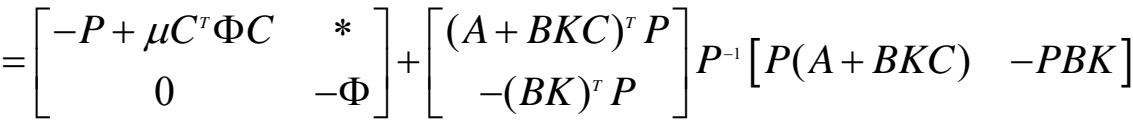

Using the Schur complement, if the following Eq. (10) is satisfied, then $\Delta V(t)<0$.

$$
\left[\begin{array}{ccc}
-P+\mu C^{T} \Phi C & * & * \\
0 & -\Phi & * \\
P A+P B K C & -P B K & -P
\end{array}\right]<0
$$

But there are nonlinear terms $P B K C$ and $P B K$ in the above Eq. (10), so it is not a LMI. Pre-multiply and post-multiply with $\operatorname{diag}\left\{P^{-1}, C P^{-1}, P^{-1}\right\}$. Define some new variables as

$$
\tilde{P}=P^{-1}, \tilde{\Phi}=\tilde{P} C^{T} \Phi C \tilde{P}, Y=K C \tilde{P}
$$

Then according to the Lyapunov stability theory for discrete-time system, system (7) is asymptotically stable if the LMI (9) is satisfied. The proof is completed.

\section{Simulation example}

Thinking about the following discrete-time systems to illustrate the scheme proposed in the paper,

$$
\begin{aligned}
x(k+1) & =A x(k)+B u(k) \\
y(k) & =C x(k) \\
\text { where } A & =\left[\begin{array}{cc}
0 & 1 \\
-1 & -2
\end{array}\right], B=\left[\begin{array}{l}
0 \\
1
\end{array}\right], C=\left[\begin{array}{ll}
1 & 0 \\
0 & 2
\end{array}\right] .
\end{aligned}
$$

First, choose $\mu=0.3$. Applying theorem (1), the feasibility problem solver function from LMI Toolbox is invoked and $t_{\min }=-0.0146<0$ is obtained, the result proves that this closed-loop system exists a group of feasible solutions:

$$
\tilde{P}=\left[\begin{array}{cc}
3.3935 & -1.9566 \\
-1.9566 & 1.8085
\end{array}\right], \quad \tilde{\Phi}=\left[\begin{array}{cc}
2.9618 & -0.5560 \\
-0.5560 & 1.0124
\end{array}\right], Y=\left[\begin{array}{lll}
0.7064 & 0.4882
\end{array}\right]
$$

At the same time, the corresponding feedback gain matrix and event-triggering matrix $\Phi$ can be gained:

$$
\Phi=\left[\begin{array}{ll}
1.8063 & 1.0939 \\
1.0939 & 0.7323
\end{array}\right], \quad K=\left[\begin{array}{ll}
0.9671 & 0.6581
\end{array}\right]
$$


The initial condition is assumed to be $x_{0}=\left[\begin{array}{ll}2 & 0\end{array}\right]^{T}$, the simulation time is chosen as $t \in[0,10]$. The initial state response curve of the closed-loop system (7) with the obtained feedback gain $K$ is shown in Figure 2.

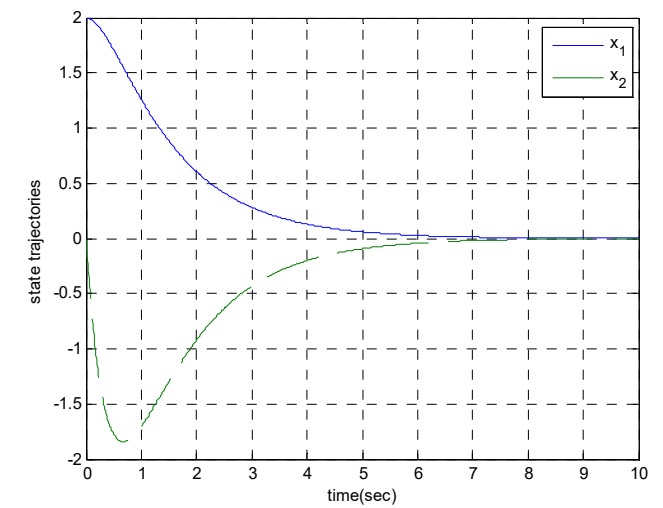

Fig.2. The initial state response curve of system

\section{Conclusion}

A novel scheme which incorporates output feedback predictive control and event-triggered communication scheme based on the networked control system with packet loss is proposed in this paper. On the one hand, the network bandwidth resources are saved. On the other hand, packet loss is compensated actively. Then, stability criterions are established which can guarantee the asymptotic stability of the closed-loop system and output feedback controller gain is obtained using the approach of a common quadratic Lyapunov and linear matrix inequality. A simulation example is given to demonstrate the effectiveness of the proposed method. The whole improvement of quality of performance and network quality of service is realized.

\section{References}

[1] Lehmann D, Lunze J. Event-based control: a state-feedback approach[J]. Automatic, 2010, 46(1): 211-215.

[2] Heemels W, Donkers M, Teel A. Periodic Event-triggered Control Based on State Feedback[C]. IEEE Conference on Decision and Control and European Control Conference, 12-15 December, 2011, Orlando, Florida: IEEE, 2011: 2571-2576.

[3] Lehmann D, Lunze J. Event-based output-feedback control[C]. 19th IEEE Mediterranean Conference on Control and Automation, Corfu, 20-23 June, 2011, Greece: IEEE, 2011: 982-987.

[4] Wang $\mathrm{X}$, Lemmon M. Event-Triggering in distributed networked control systems[J]. IEEE Transactions on Automatic Control, 2011, 56(3): 586-601.

[5] Guoping Liu, Xiuxia Yin, Rees D. Design and stability criteria of networked predictive control systems with random network delay in the feedback channel[J]. Systems, Man, and Cybernetics, Part C: Applications and Reviews, IEEE Transactions on, 2007, 37(2): 173-184.

[6] Guoping Liu, Xiuxia Yin, Chen J. Networked predictive control of systems with random network delays in both forward and feedback channels[J]. IEEE Transactions on Industrial Electronics, 2007, 54(3): 1282-1297.

[7] Wenshan Hu, Guoping Liu, Rees D. Event-driven networked predictive control[J]. IEEE Transactions on Industrial Electronics, 2007, 54(3): 1603-1613.

[8] Xiuxia Yin, Dong Yue, Chen Peng. Model based event triggered predictive control for network systems with data dropout[J]. Siam Journal on Control \& Optimization, 2016, 54(2): 567-586. 